\title{
Realistic following behaviors for crowd simulation
}

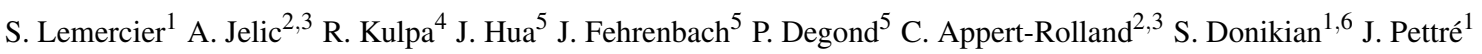 \\ ${ }^{1}$ INRIA Rennes - Bretagne Atlantique, Campus de Beaulieu, 35042 Rennes, France \\ ${ }^{2}$ Univ. Paris-Sud, Laboratoire de Physique Théorique, Bât. 210, F-91405 Orsay Cedex, France \\ ${ }^{3}$ CNRS, LPT, UMR 8627, Bât 210, F-91405 Orsay Cedex, France \\ ${ }^{4}$ M2S - MimeTIC - University Rennes 2, Rennes, France \\ ${ }^{5}$ Institut de Mathématiques de Toulouse, Université Paul Sabatier, 118 route de Narbonne, Toulouse, France \\ ${ }^{6}$ Golaem S.A.S.
}

\begin{abstract}
While walking through a crowd, a pedestrian experiences a large number of interactions with his neighbors. The nature of these interactions is varied, and it has been observed that macroscopic phenomena emerge from the combination of these local interactions. Crowd models have hitherto considered collision avoidance as the unique type of interactions between individuals, few have considered walking in groups. By contrast, our paper focuses on interactions due to the following behaviors of pedestrians. Following is frequently observed when people walk in corridors or when they queue. Typical macroscopic stop-and-go waves emerge under such traffic conditions. Our contributions are, first, an experimental study on following behaviors, second, a numerical model for simulating such interactions, and third, its calibration, evaluation and applications. Through an experimental approach, we elaborate and calibrate a model from microscopic analysis of real kinematics data collected during experiments. We carefully evaluate our model both at the microscopic and the macroscopic levels. We also demonstrate our approach on applications where following interactions are prominent.
\end{abstract}

Categories and Subject Descriptors (according to ACM CCS): Computer Graphics [I.3.7]: Animation—; Simulation and Modeling [I.6.4]: Model Validation and Analysis—; Simulation and Modeling [I.6.5]: Model Development-;

\section{Introduction}

Typical large-scale patterns of pedestrians are formed by moving crowds. These structures are unconsciously selforganized by people in the crowd, and systematically emerge under specific traffic conditions. Their cause is the combination of the numerous interactions that pedestrians have at a local scale. One goal of microscopic crowd simulation is to reproduce these emergent phenomena. Patterns emerge in simulations of pedestrian traffic under specific setups, as for real patterns under specific conditions. This emergence entirely depends on the numerical model of local interactions between agents. Reproducing realistic patterns is thus a promising path to validate crowd simulation models.

In this paper, we are interested in the stop-and-go waves that emerge from unidirectional pedestrian traffic. They are frequently observed when people walk in corridors or when they queue. In such a situation, pedestrians are forced to temporarily follow each other in order to reach their individual destination. We believe that these following interactions are the main cause of the emergence of stop-and-go waves.

Our motivation is first to explore following behaviors in crowd simulation. This type of interaction was relatively neglected compared to collision avoidance or walking in groups. Second, we aim at elaborating a microscopic model of following interactions which would both enable synthesizing realistic individual trajectories with interactions at a local scale, as well as simulating the emergence of realistic patterns of pedestrians at a larger scale. Realism here means a direct comparison between simulations and real examples.

Our approach is an experimental one. We assume that following someone mainly consists in constantly adapting his own speed with respect to the motion of the followed walker. Under this hypothesis, our problem can be reduced to the 1dimensional control of tangential velocity. We first propose to observe how humans perform such motion control. For best accuracy, we choose to motion capture several participants following each other during laboratory experiments. 


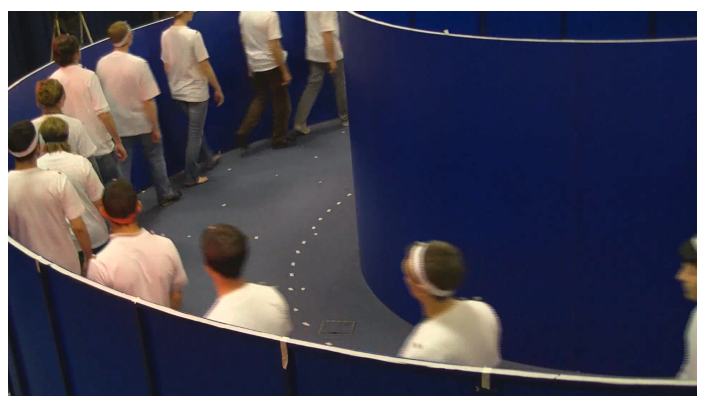

Figure 1: Experiments on 1-D traffic: participants were asked to walk without overtaking each other. At the microscopic scale, we observe how humans adapt their motion to follow someone. At the macroscopic scale, we observe the emergence of stop-and-go waves from such interactions: the trough of such a wave is visible on picture's leftest part.

We design protocols to study 1-D traffic under changing conditions of density. We are able to observe emergent stopand-go waves from this protocol. We extract their properties (propagation speed through traffic as well as damping in time). Our data are precise enough to enable accurate calibration of a microscopic model of following behavior derived from car traffic simulation. We comparatively study the emergence of stop-and-go waves in reality and in simulations based on our new model.

Our contributions are:

1. an experimental study on following behaviors. Using a carefully designed protocol, we motion-captured up to 28 participants following each other. We obtained a highprecision kinematics dataset that both describes individual motions as well as emergent stop-and-go waves.

2. a numerical model of following behavior. Inspired by the analogy with car traffic, we derived our model from the Aw-Rascle car traffic model [AKMR02].

3. a dedicated calibration technique. Calibration is implicitly equivalent to an evaluation of the model at the microscopic scale. We also show the capacity of the model to simulate the emergence of stop-and-go waves at a macroscopic scale. This 2-level evaluation is an opportunity to discuss micro-macro coupling problems.

The remaining of this paper is organized as follows. In Section 2 we situate our work in the fields of crowd simulation, car traffic simulation and experiments on pedestrian traffic. Section 3 describes our experiments on 1-D pedestrian traffic. Microscopic and macroscopic analysis of experimental kinematics data is performed in Section 4. Next, in Section 5 we introduce our model of following interactions. We finally calibrate and evaluate our model: we compare it to real data as well as to other models in Section 6 and demonstrate it over examples in Section 7 before Discussion and Conclusion.

\section{Related Work}

Crowd simulation is a very active topic and many different kind of models have been proposed to perform simulation: cellular automatons [Sch01], social forces [HFV00], continuum flow [TCP06], agent-based [ST07], geometrical [GCK $\left.{ }^{*} 09\right]$ or synthetic vision-based models [OPOD10]. All of these models consider collision avoidance as the only way of physical interaction between pedestrians.

Following interaction has been more rarely addressed. In computer animation, such interaction is used to simulate social groups. Groups may follow a leader [LJC01]. Musse et al. [MT01] define rules to model flocking formation in groups. Using a social forces based-model to simulate virtual crowds, Braun et al. [BMdOB03] implement an altruism force to urge pedestrians on to walk together. Pelechano et al. [PAB07] use areas of influence to model unidimensional traffic. A more recent velocity-based approach reproduces realistic formations of small groups [KO10].

However, walking in groups is often closer to flocking than strictly following a moving target, such as when walking in Indian file. Loscos et al. [LMM03] propose different reactions for a following behavior depending on the distance and the behavior of a leader. More than for flocking behavior [Rey87], Reynolds also proposes a leader following implementation [Rey99] on which the follower targets a point offset slightly behind the leader and shadows this target by matching its speed and heading.

Several experiments have been carried out to study pedestrian behavior. Daamen and Hoogendoorn [DH03] performed a set of experiments to observe pedestrians in 1directional and 2-directional traffic, crossing traffic and at bottlenecks. They could notably observe lane formation, and sliding waves front in crossing flows. Yamori [Yam98] studied the formation of lanes at a large crosswalk. Kretz et al. [KGS06] studied pedestrian behavior in bottlenecks. More in the context of 1-dimensional studies, Seyfried et al. [SSKB05] established a relation between pedestrian speed and density called fundamental diagram observing people who had to walk in single lane in a looped corridor. They also observed the cultural modulation of fundamental diagrams in [CSC09] by comparing the traffic of German and Indian walkers.

Finally, studies on human motion have been carried out in biomechanics and cognitive science. Lee [Lee76] introduces a theory of visual control of braking in the context of car driving. He uses the optic variable $\tau$ to specify the driver's time to collision. $\dot{\tau}$, the first derivative of the optic variable, determines if the deceleration is strong enough to avoid a collision with the obstacle. Yilmaz et al [YW95] carried out an experiment which supports Lee's $\tau$ strategy. However they notice that humans can make predictions only when time-to-collision is less than 2-3 seconds. Fajen [Faj07] goes further by proposing an affordance-based approach using the $\tau$ theory, considering the subject's braking capabilities. In 
the case of pedestrians following each others we experimentally measured that time-to-collision is, most of the time, much higher.

In transportation science, Aw et al. [AKMR02] propose a model of vehicular traffic on which the tangential acceleration $a$ is obtained as follows:

$$
a=C \frac{v_{i+1}-v_{i}}{\left(x_{i+1}-x_{i}\right)^{\gamma+1}}+A \frac{1}{T_{r}}\left[v\left(\frac{\Delta X}{x_{i+1}-x_{i}}\right)-v_{i}\right]
$$

where $x_{i}(t), v_{i}(t)$ are location and speed of vehicle $i$ at time $t \in \Re^{+}, \Delta X$ is the length of the car, $C>0, A>0, \gamma \geq 0$ and $T_{r}$ are constants. Considering the homogeneous case $A=0$, which removes the relaxation term of equation 1 , this model is similar to Lee's one. However, here, the acceleration is not function of $\tau$ but of its inverse.

We notice that only few models for following behavior have been proposed and none of them is evaluated in comparison with real human behavior. Our goal is to elaborate a model of following behavior which guarantees a high level of realism. We propose experimental protocols to compose a reference kinematics dataset. We use optoelectronic motion capture to obtain high accuracy kinematics data whereas video-based techniques were previously employed. To our knowledge, our dataset is the most accurate measurement of pedestrian traffic performed so far. Such accuracy allows us to detail the microscopic interactions between people following each other by analyzing their individual motion signals (position, speed and acceleration) with yet unreached precision. It also allows us to directly calibrate a microscopic simulation model on individual data, and to access a distribution of parameters from the observed population. However we also design our experiments to observe macroscopic phenomena. From Aw et al. [AKMR02] model for road traffic, we verify their hypothesis in pedestrian traffic and formulate a new model of interactions adapted to crowd simulation.

\section{Experiments on 1D traffic}

Objective. The objective of the proposed experiment is to study the kinematics of human locomotion in the task of following another human. Two questions are raised. First, at a microscopic level, how do humans control their locomotion when following someone? Second, at a macroscopic level, do some stop-and-go waves emerge from such interactions?

Task. Participants were asked to walk in line on a circular path delimited by walls, following one behind the other at natural speed "as if they were walking alone in the street". They were not allowed to overtake each other.

Population. 28 volunteers (20 males and 8 females) participated to our experiment. They gave their participation agreement by signing an informed consent. The study was conformed to the Declaration of Helsinki. Participants were all naïve with respect to the purpose of our experiment. They were recruited through advertising.
Experimental conditions. We studied the effect of pedestrian density on the global traffic conditions (flow speed) and on the characteristics of macroscopic emergent phenomena. Table 1 summarizes the studied densities. In addition, to avoid some bias resulting from initial conditions, we considered two ways of starting trials: participants were uniformly spaced out before starting walking or packed together, letting some experimental space empty. These conditions are illustrated in Figure 2.

\begin{tabular}{|c|c|c|}
\hline $\begin{array}{c}\text { number of } \\
\text { participants }\end{array}$ & $\begin{array}{c}\text { circle } \\
\text { perimeter }(\mathrm{m})\end{array}$ & $\begin{array}{c}\text { density } \\
\left(p . m^{-1}\right)\end{array}$ \\
\hline 8 & 25.76 & 0.31 \\
16 & 25.76 & 0.62 \\
24 & 25.76 & 0.93 \\
16 & 15.08 & 1.06 \\
28 & 25.76 & 1.09 \\
20 & 15.08 & 1.33 \\
21 & 15.08 & 1.39 \\
24 & 15.08 & 1.59 \\
28 & 15.08 & 1.86 \\
\hline
\end{tabular}

Table 1: List of studied densities. By combining the number of participants in our study and the perimeter of the circular path they followed we could study 9 different conditions of density ranging from 0.31 to $1.86 \mathrm{p.m} \mathrm{m}^{-1}$
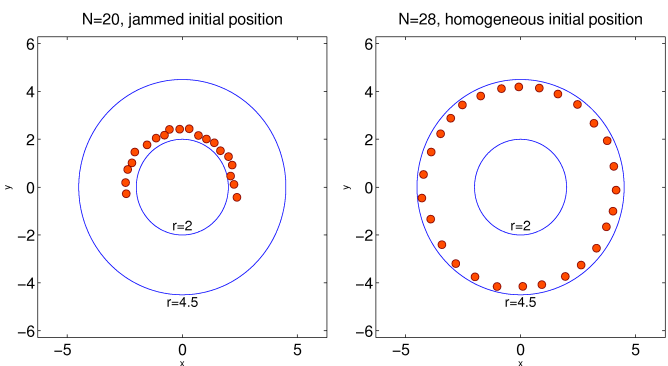

Figure 2: Illustrations of experimental conditions: number of participants, path length and initial positions. The experimental system is represented from top. The two large circles correspond to the circular walls physically delimiting the system. The smaller circles represent participants at their initial position. Left: 20 participants are initially packed together along the inner circle. Right: 28 participants are uniformly spaced out along the outer circle.

Experimental apparatus. In order to study various conditions of density, we both played on the number of participants walking in a given trial as well as the length of the path they were walking along. To avoid some side effects, participants followed a circular path delimited by walls as illustrated in Figure 1. They were asked to follow the inner wall or the outer wall of the dedicated structure which resulted into two possible path lengths: 
- the inner circle path was $2.4 m$ of radius, i.e., $15.08 m$ long,

- the outer circle path was $4.1 \mathrm{~m}$ of radius, i.e., $25.76 \mathrm{~m}$ long.

Experimental plan. We successively asked 8, 16, 24, 28 people to walk along the outer circle. Initial conditions (packed together or uniformly spaced out) were alternatively used. We successively asked 16, 20, 21, 24 and 28 people to walk along the inner circle. Again, the same initial conditions were alternatively used. This resulted into the following average density of participants: $0.31,0.62,0.93,1.06$, $1.09,1.33,1.39,1.59$ and 1.86 p.m ${ }^{-1}$. For each condition (density and dispersion), a various number of repetitions were carried out ( 2 to 8 ). Indeed, we did not try to obtain an equilibrated experimental plan: we combined experimental constraints to maximize the range of density covered by our experiment and repeated measures when interesting phenomena (stop-and-go waves) occurred. The overall experiment lasted 2 hours, and 52 trials of 1 minute each were carried out.

Collected Data. Participants' trajectories were measured using a Vicon MX-40 motion capture system with 12 cameras running at $120 \mathrm{~Hz}$. Each participant was wearing a white top and four markers: one at the left shoulder, two at the right one and one on the head. Data reconstruction is described in $\left[\mathrm{LMM}^{*} 11\right]$.

\section{Experimental Results}

The global motion of each participant is estimated to be the one of the barycenter of the four markers they were wearing. We filter the resulting global trajectories: we apply a lowpass Butterworth filter with a cut-off frequency of $0.1667 \mathrm{~Hz}$ to remove the effect of steps. For convenience, and because the path followed by participant is a circle, we compute trajectories in a cylindrical coordinate system, which center coincides with the one of the experimental circular corridor. Thus, the position of participant $i$ at time $j$ is described as follows:

$$
\mathbf{p}_{i, j}^{c y l}=\left[\theta_{i, j} r_{i, j} z_{i, j}\right]
$$

By computing the actual average radius of the circular path that participants followed $r_{\text {mean }}$, we approach the global 1-D linear motion (in meters and seconds) as follows:

$$
\left\{\begin{aligned}
p_{i, j} & \approx \theta_{i, j} * r_{\text {mean }} \\
v_{i, j} & \approx \dot{\theta}_{i, j} * r_{\text {mean }} \\
a_{i, j} & \approx \ddot{\theta}_{i, j} * r_{\text {mean }}
\end{aligned}\right.
$$

\subsection{Microscopic Analysis}

Microscopic analysis inspects individual trajectories to understand how humans control their locomotion when following someone. We search for cross-correlations between oneself motion signals (position, speed, acceleration) and relative motion signals (relatively to the followed human: distance, speed, time to contact, etc.). We follow hypotheses formulated in previous work and compare them. Our
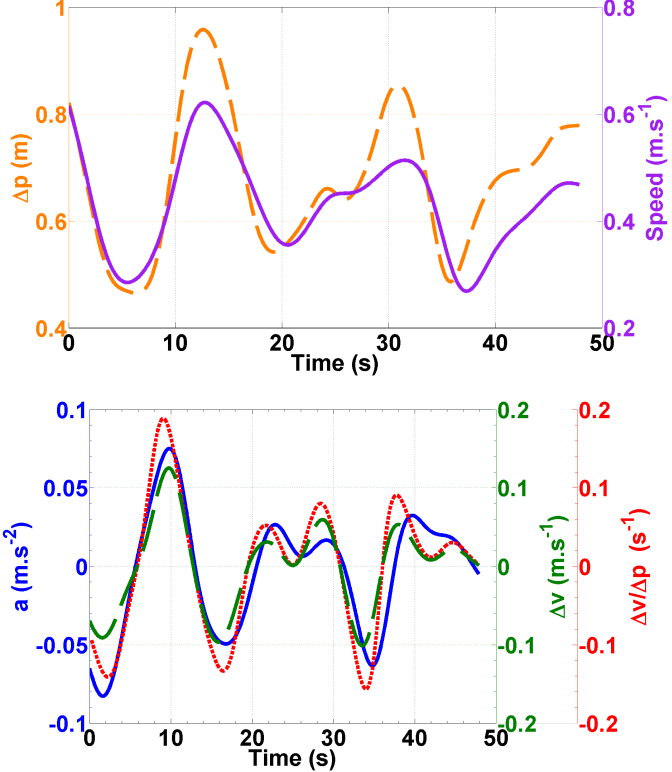

Figure 3: Motion signals for a given participant during a complete experimental trial. Top: self-speed (orange) and distance to target (purple). Bottom: self-acceleration (blue), relative speed to the followed participant (green) and relative speed over distance to target (red).

findings are illustrated in Figure 3 from a representative example. Distance is denoted $\Delta p=\left(p_{i+1, j}-p_{i, j}\right)$ and relative speed $\Delta v=v_{i+1, j}-v_{i, j}$. In this example, we observe a strong cross-correlation between speed and distance signals (Figure 3, upper plot), a stronger correlation between acceleration and relative speed, and finally, an even stronger correlation between acceleration and relative speed over distance $\Delta v / \Delta p$ Figure 3 , bottom plot). Figure 4 displays the mean cross-correlation values that were obtained for all the experimental trials, grouped by condition of density and for all the studied pairs of signals. Correlation between acceleration and relative speed or relative speed over distance (respectively $a \star \Delta v$ and $a \star \Delta v / \Delta p$ ) both reach highest possible values, up to 0.74 . Cross-correlation is the highest when density is between 1.0 and 1.9 ped.m $\mathrm{m}^{-1}$.

We interpret results as follows. A model which controls the tangential acceleration as a function of relative speed over distance is more coherent with experimental data. Two reasons may explain the low correlation scores - whatever the considered signals - obtained for low and very high densities. At low densities, motion is less constrained and participants walk with higher variance. At high densities, the quality of motion captures is sometimes mediocre due to numerous markers occlusions in raw motion data. 


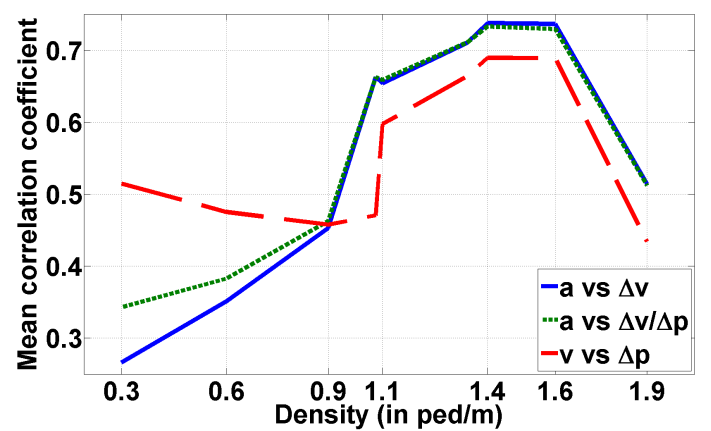

Figure 4: Mean cross correlation between pairs of real signals grouped by density. Blue: mean $((a \star \Delta v)(t))$. Green: mean $((a \star \Delta v / \Delta p)(t))$. Red: mean $((v \star \Delta p)(t))$.

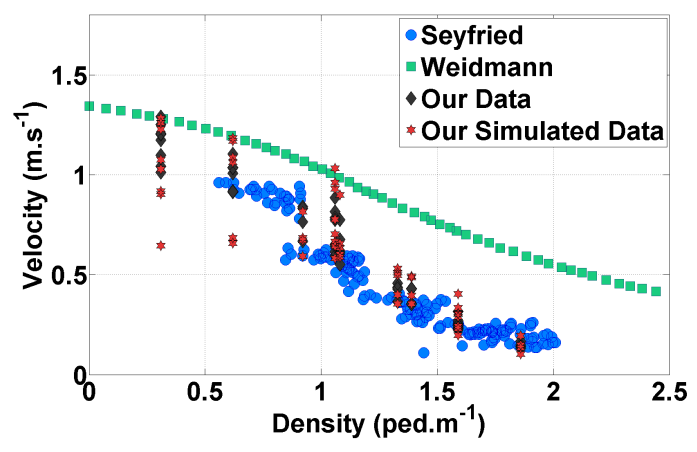

Figure 5: Fundamental Diagram. Mean speed of participants with respect to global density. Values obtained from our experiments are displayed with those from Seyfried and Weidmann.

\subsection{Macroscopic Analysis}

The goal of macroscopic analysis is to describe the observed global traffic conditions as well as the emergent stop-and-go waves. Global traffic conditions are described from the fundamental diagram of participant flow as shown in Figure 5. This diagram emphasizes the relationship between participants mean speed and mean density (cf. Table 1 for the list of studied densities). Each point corresponds to a different trial: the speed is averaged over all participants and over the whole duration of a trial (1 minute). We compare our results with previous fundamental diagrams (as well as simulated data, as presented in Section 6). As expected, velocity decreases when density increases and our results are consistent with independent measurements performed by Seyfried et al. [SSKB05]. There is a discrepancy with the Weidmann [Wei93] fundamental diagram, but those measurements were obtained by combining 25 experiments performed in different settings (not necessarily with one-dimensional flows).

The speed of a pedestrian actually varies during a trial. Figure 6 shows spatiotemporal plots describing all the partic-
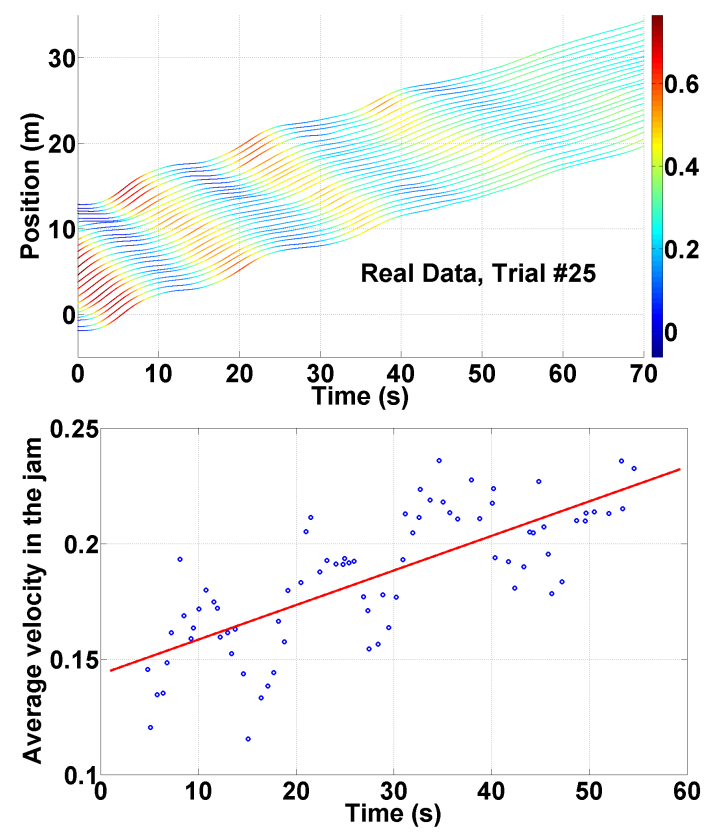

Figure 6: Experimental data for trial \#25. Top: individual 1-D position with respect to time, with color as a function of the speed. Bottom: mean speed of participant going through the jam with linear regression.

ipants' individual positions colored by speed. The formation of some spatio-temporal waves which propagate counterflow are clearly observed. These are characterized by successive occurrences of stages with relatively high speeds and stages with slower speeds. Waves damp in time and the traffic converges to a laminar flow.

We are able to deduce the wave propagation speed by tracking the troughs of the wave, i.e., jams. Jams occur where participants reach a local minimum speed. A wave is the propagation of this local minimum and thus, of the jam, from participant to participant. A jam is detected when aparticipant speed is below a certain threshold $c . \bar{v}$, where $\bar{v}$ is the mean speed for all the participants and for the whole duration of a trial (excluding the initial transient). $c$ is a constant which typically takes value between 0.8 and 1 , and which is manually tuned depending on each trial. The propagation speed is deduced from the successive positions in time of the front and the end of the jam (respectively where participants decelerate and re-accelerate before and after the jam). Example values are provided in Table 2.

Waves damping is characterized from the speed of participants while they pass through the jam. This speed increases bit-by-bit and becomes closer to the speed of the laminar flow. Figure 6, bottom, shows successive measures of the lowest participants speeds for trial \#25. We can deduce a 


\begin{tabular}{|c|c|c|c|c|c|}
\hline $\begin{array}{c}\text { trial } \\
\text { id } \\
\#\end{array}$ & $\begin{array}{c}\text { density } \\
p . m^{-1}\end{array}$ & $\begin{array}{c}\bar{v} \\
m . s^{-1}\end{array}$ & $\begin{array}{c}\text { front } \\
\text { velocity } \\
m . s^{-1}\end{array}$ & $\begin{array}{c}\text { end } \\
\text { velocity } \\
m . s^{-1}\end{array}$ & $\begin{array}{c}\text { damping } \\
m . s^{-2}\end{array}$ \\
\hline$\# 13$ & 1.86 & 0.172 & 0.654 & 0.611 & -0.0005 \\
$\# 25$ & 1.59 & 0.304 & 0.691 & 0.608 & 0.0015 \\
$\# 32$ & 1.59 & 0.235 & 0.574 & 0.512 & -0.000006 \\
$\# 35$ & 1.59 & 0.238 & 0.683 & 0.673 & 0.0007 \\
$\# 38$ & 1.39 & 0.347 & 0.632 & 0.614 & -0.0003 \\
\hline
\end{tabular}

Table 2: Result of macroscopic analysis of trials \#13, \#25, \#32, \#35 and \#38

damping velocity by linear regression of these successive measures. The slope of the regression is $0.0015 \mathrm{~m} . \mathrm{s}^{-2}$.

We are now able to provide some detailed properties of the emerging stop-and-go waves which propagate through 1-D traffic. General conclusions are not easy since the emergence of waves and their properties are partly chaotic. For example, no wave is clearly detected when density is below 1 p. $m^{-1}$, or only very local ones that propagates through few participants. Between 1 p. $\mathrm{m}^{-1}$ and $1.6 \mathrm{p} . \mathrm{m}^{-1}$ waves are observed most of the time, but not always. However, when appearing, they systematically propagate counterflow with similar speed. Damping is apparently a more complex phenomenon. Under similar conditions, we observed damping waves, but also reappearing waves, or even instable waves with increasing amplitude. Damping, as illustrated in Figure 6 , was however our most frequent observation.

\section{A realistic model of following behavior}

Inspired by the results of our microscopic analysis and by the Aw-Rascle model [AKMR02] presented in section 2, we model the following behavior by controlling the instantaneous tangential acceleration $a(t)$ :

$$
a(t)=C \cdot \Delta v(t+\tau) \cdot \rho^{\gamma}(t)
$$

where $C$ is a constant, $\Delta v(t)$ the instantaneous speed of the followed target relatively to the follower, $\tau$ a time delay, and $\rho$ the local density $(\rho=1 / \Delta p)$, which is simply computed as the inverse of the distance to the target.

Compared to the original model by Aw and Rascle [AKMR02], the main differences are that we do not consider the relaxation term and delay the relative speed one. On the analysis point of view, we actually observe a temporal shift between $a(t)$ and $\Delta v(t)$ signals as illustrated in Figure 7. On the contrary, the the density term $\rho(t)$ is not delayed. This delay $\tau$ is also meaningful in terms of human perception. Indeed, the perception of speed is made from a successive perception of distances in time and this integration process introduces a delay while the perception of distance is instantaneous.

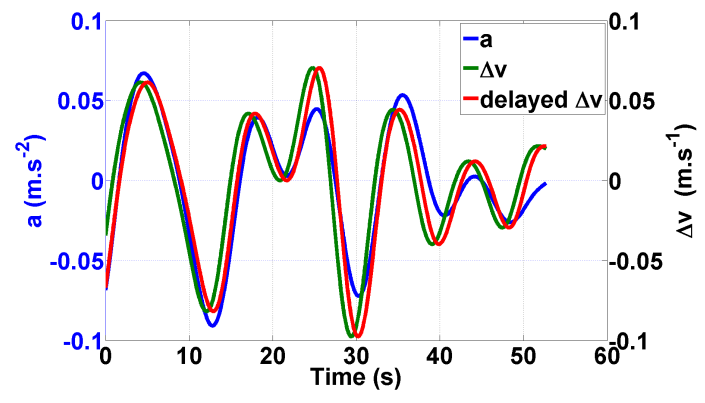

Figure 7: Acceleration signal superimposed with relative speed signal. Blue: a. Green: $\Delta v$. Red: $\Delta v$ with $\tau$ delay: one can visually note that cross-correlation is increased when the relative speed signal is delayed

\section{Evaluation}

\subsection{Microscopic Calibration}

We calibrate the three model parameters $C, \gamma$ and $\tau$ from real data using a specific calibration technique described below:

1. Data smoothing. We compute $\bar{a}, \overline{\Delta v}$ and $\bar{\rho}$ averaged over a moving time-window $W: W=\left[t_{0}, t_{0}+T\right]$, where $t_{0}$ : $0 \rightarrow \pi-T$ ( $\pi$ the duration of the experimental trial and duration $T$ is empirically set to $T=6.67 \mathrm{~s}$.).

2. $\tau^{*}$ estimation. $\tau^{*}$ is the estimated time shift in real data between $a(t)$ and $\left.\Delta v(t): \tau^{*}=\arg \max (a(t) \star \Delta v(t+\tau))\right)$, where $t \in W$ and $\tau \in[-3+2 s$. $]$ and $\star$ is the crosscorrelation.

3. Data validity domain. We estimate that the considered pedestrian complies with the model (4) in the subwindow $W$ if the following conditions are satisfied:

a. the actual $\tau^{*}$ delay is positive and less than $3 \mathrm{~s}$, since negative delays are irrelevant to our model and a delay at an upper bound of the search interval is probably not optimal,

b. the cross-correlation between $a$ and $\Delta v$ delayed by $\tau^{*}$ is above a threshold, empirically set at 0.6 in our study.

If both the conditions a) and b) described above are satisfied, the corresponding data are considered to be valid with respect to our model. In this case, the constant $C^{\prime}=\|a\| /\|\Delta v\|$ is then assumed to be the factor between $a$ and $\Delta v$ with $\tau^{*}$ delay.

4. In principle, we should have $C^{\prime}=C \cdot \bar{\rho}^{\gamma}$ for a suitable value of $\gamma$. To estimate the parameter $\gamma$ we plot for all the valid data in our experiments the quantity $C^{\prime}$ vs $\bar{\rho}$ and fit a power law by linear regression on the logarithms of these quantities. The result is shown in Figure 8, bottom.

The optimal parameter is $\gamma=-0.514$, rounded to $\gamma=$ -0.5 . Constant $C$ is then defined on each time-window $W$ by $C=C^{\prime} / \bar{\rho}^{-0.5}$.

The histograms of the obtained values for $\tau$ and $C$ are shown in Figure 8. We calibrate our model over a subset of 


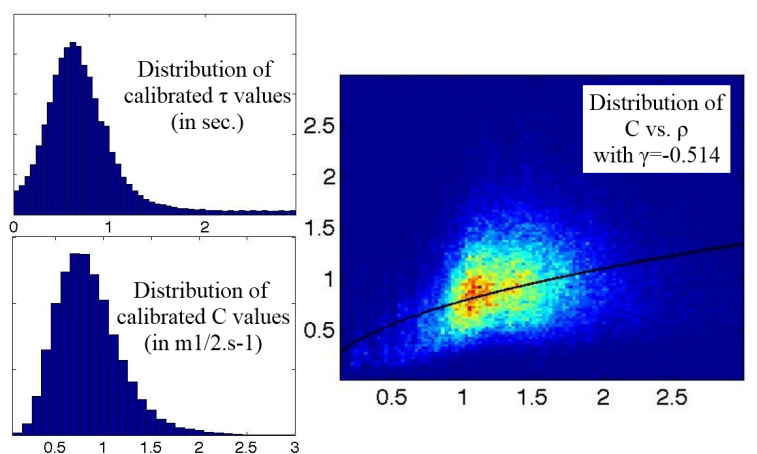

Figure 8: Top left: Distribution histogram of $\tau^{*}$ delay (median delay: $0.658 \mathrm{~s}$, standard deviation 0.45s). Bottom left: Distribution histogram of $C$ constant (median value: $0.809 m^{1 / 2} s^{-1}$, standard deviation $\left.0.355 m^{1 / 2} s^{-1}\right)$. Right: Joint distribution of $\bar{\rho}$ and $C^{\prime}$. The fit of a power law provides the optimal $\gamma$ parameter.

experimental data considered to be valid according to calibration step 3. This subset represents $77.4 \%$ of all our experimental data. This high proportion corroborates that the proposed model (4) is coherent with microscopic analysis of our experimental data. Deeper analysis shows that this proportion is higher when global density is above 1 ped.m $\mathrm{m}^{-1}$, which also corroborates the results illustrated in Figure 4). Next section explores model validity at the macroscopic scale: we compare fundamental diagrams between real and simulated data as well as the properties of emerging stopand-go waves.

\subsection{Macroscopic evaluation}

The microscopic calibration presented in previous Section implicitly validates our model at this scale. We here explore validation at a macroscopic scale. We first compare the relation between density and flow speed between real and simulated data. We prepare 52 simulation setups that reproduce the initial experimental conditions of our 52 trials (participants positions and speeds). Model parameters are distributed according to the results of calibration (Figure 8). We run each simulation for 1 minute (simulation time) and measure the mean speed with respect to the density. Figure 5 shows the fundamental diagram resulting from simulations in comparison with real observations. Results confirm the adequacy between the calibrated model and real data, especially for the densities above 1 ped. $\mathrm{m}^{-1}$. Actually, Wilcoxon signed-rank tests on measurements grouped by density reveal that simulation and real data are all similar, but for the 0.31 ped. $\mathrm{m}^{-1}$ situation.

In a second step, we look for the emergence of stop-andgo waves through simulated data under similar conditions than real observations. A direct confrontation with real data
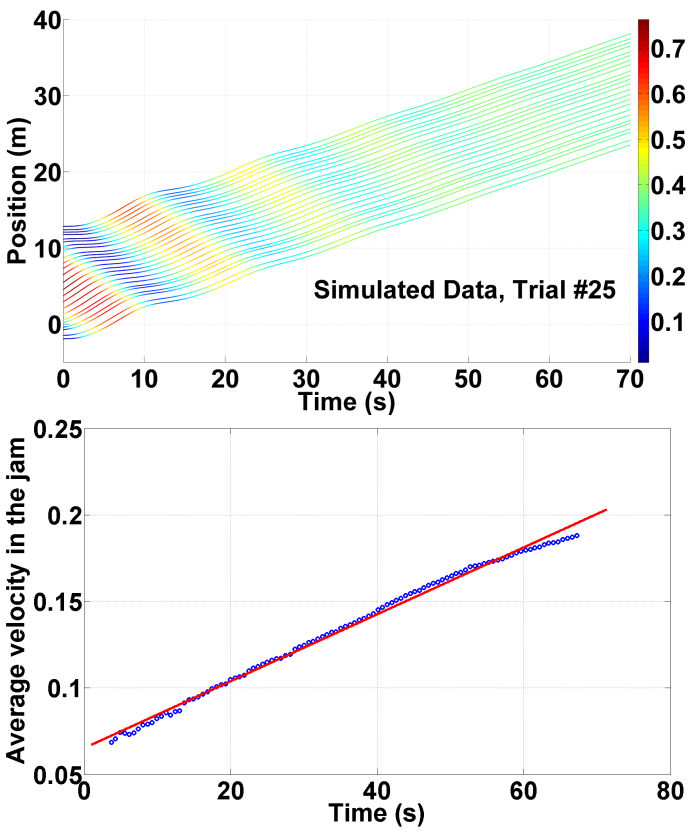

Figure 9: Simulation is here initialized from the conditions of a specific experimental trial: id \#25 (24 participants, inner circle, average density 1.59 ped. $\mathrm{m}^{-1}$, homogeneous starting). Both plots can be directly compared to those in Figure 6. Top: individual trajectories. Bottom: mean speed of pedestrian going through the jam with linear regression.

\begin{tabular}{|c|c|c|c|c|c|}
\hline $\begin{array}{c}\text { simul. } \\
\text { id } \\
\#\end{array}$ & $\begin{array}{c}\text { density } \\
p . m^{-1}\end{array}$ & $\begin{array}{c}\bar{v} \\
m . s^{-1}\end{array}$ & $\begin{array}{c}\text { front } \\
\text { velocity } \\
m . s^{-1}\end{array}$ & $\begin{array}{c}\text { end } \\
\text { velocity } \\
m . s^{-1}\end{array}$ & $\begin{array}{c}\text { damping } \\
\text { (slope) } \\
m . s^{-2}\end{array}$ \\
\hline$\# 13$ & 1.86 & 0.168 & 0.576 & 0.549 & 0.0004 \\
$\# 25$ & 1.59 & 0.270 & 0.748 & 0.718 & 0.0019 \\
$\# 32$ & 1.59 & 0.255 & 0.770 & 0.733 & 0.0003 \\
$\# 35$ & 1.59 & 0.223 & 0.792 & 0.762 & 0.0007 \\
$\# 38$ & 1.39 & 0.375 & 0.503 & 0.496 & -0.0013 \\
\hline
\end{tabular}

Table 3: Macroscopic analysis results for simulations under the initial conditions of trials \#13, \#25, \#32, \#35 and \#38.

is not possible in that way. Detailed analysis reveals instabilities in simulations using calibrated parameters. We empirically find out that combined values between $C$ and $\tau$ lead to such instabilities, and that stability is obtained when C. $\tau<0.5$. We are currently exploring this problem of transition from microscopic to macroscopic scales and how they are coupled. Nevertheless, in the following analysis, we increase the value of $C$ and lower the one of $\tau$ compared to calibration. We thus stay in the stable domain of our numerical model. We take a unique parameter setup for all the simulated agents to avoid complex interactions. We recalibrate the model applying a the least-squares method on participants' speed. We obtain $C=1.3$ and $\tau=0.1 s$. Fig- 
ure 9 shows the results of a simulation under the conditions of experimental trial \#25 (introduced in Figure 6 which can be directly confronted to Figure 6). Trajectories are colored with respect to the amplitude of the instantaneous speed to emphasize the stop-and-go waves. Their propagation is well reproduced. Mean velocity is $0.304 \mathrm{~m} \cdot \mathrm{s}^{-1}$ in real data and $0.270 \mathrm{~m} \cdot \mathrm{s}^{-1}$ in simulations (cf. Tables 2 and 3 ). The propagation speed of the stop-and-go wave is also compared and similar results are obtained. In this specific example, the damping effect is correctly reproduced as well. More generally, comparison between Table 3 and Table 2 shows that the emergence of waves and their propagation is correctly reproduced in simulations. The damping effect, which was analyzed to be behave in a more chaotic manner, is not correctly reproduced for all trials.

\subsection{Comparison with other models}

Our model is designed from a microscopic perspective. Microscopic analysis suggests that our model better matches microscopic data than velocity control to maintain respectful distance between agents. Reynolds's proposed such velocity control in [Rey99]: follower agent shadows a virtual point placed at a distance behind the followed agent. Trajectories simulated base with Reynolds's model under the experimental conditions are shown in Figure10. Such a model fails toreproduce wave propagation at the macroscopic level.

We also compared our results with avoidance models. Indeed, temporarily following someone because overpassing is not doable can be considered as collision avoidance. The Helbing's social force model [HFV00] is able to simulate people queuing by simply projecting all the acceleration forces on the axis of the queuing lane. People keep separated by repulsive forces that are function of distance. Resulting trajectories are shown in Figure10. We confirm that this avoidance model is able to simulate 1D traffic with stop and go waves emerging, but fails to reproduce the aspect of real waves as well as the damping effect. Finally note that our microscopic analysis suggests that the distance parameters of these two models should be calibrated again depending on density factors or velocity factors.

We extend our model formulation to 2D motion control to perform large corridor scenarios (cf. Section 7.2 and companion video). We model the pedestrian traffic similarly to a multiple lanes highway traffic, with the difference that lanes are not physically defined. In our simulations, the longitudinal velocity component (i.e., along the corridor axis) is controlled using our model: each agent follows the agent immediately in front of him. The lateral velocity component is controlled by a simple rule: if there is enough room on the side of the followed agent (no wall and no other agent too close), the lateral velocity is increased toward the corresponding side to enable overpassing. This extension is compared with Helbing [HFV00] (with unconstrained acceleration forces), RVO2 [GCC*10] and Tangent [POO*09] mod- els. None of these models reproduces stop-and-go waves in corridor traffic simulation. However, we obtain various distribution of pedestrians: whereas Helbing's model results into uniform distribution, $\mathrm{RVO} 2$ results into abnormal distributions with aggregations and empty spaces. One future work direction is to compare with latest versions of Helbing's model [JHS07] or to add a delay to these models.

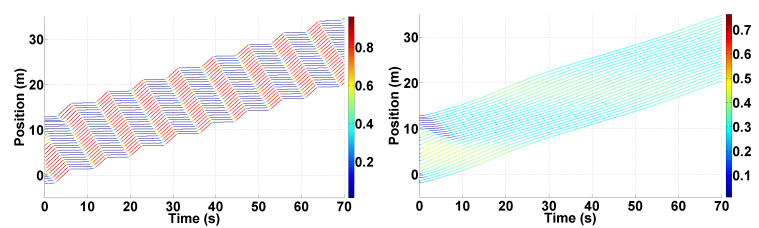

Figure 10: Helbing (left) and Reynolds (right) simulated trajectories for trial id \#25.

\section{Results}

\subsection{Parameters role}

The proposed follower model is controlled from three parameters: $C, \tau$ and $\gamma$. The relation between these parameters and the local behavior of a virtual pedestrian is relatively intuitive. $C$ changes the dynamic of the pedestrian by directly scaling the acceleration amplitude, $\tau$ is explicitly the pedestrian reaction time, and finally $\gamma$ changes the pedestrians inbetween distance by increasing the amplitude of accelerations while this distance becomes lower. Relating parameters values with macroscopic phenomena is less intuitive. We empirically determine their influence. $C$ mainly plays on wave propagation speed: the lower $C$ the slower the wave speed propagation, and inversely. Independent changes of $\tau$ have an effect on the damping of stop-and-go waves. The higher $\tau$ the longer waves last and the less they damp. All these effects are illustrated in Figure 11. Simulation under the conditions of trial \#25 are run again while $C$ and $\tau$ values are changed.

\subsection{Applications}

We illustrate our results over two different scenarios as shown in Figure 12 and companion video. First scenario strictly matches the conditions of our experimental study. We simulate numerous virtual pedestrians walking in Indian file. Simulation controls the motion of the head of the queue where a virtual checkpoint is placed: the pedestrian in first place is periodically allowed to continue his way. Thus, we generate waves that propagate along the queue. A winding path line ensures an easy view of the whole.

The second example simulates traffic in a corridor as explained in Section 6.3. The second scenario opens interesting perspectives. It shows that complex behaviors can be obtained by combining various control laws for speed and orientation control. It opens the application range of our model. 

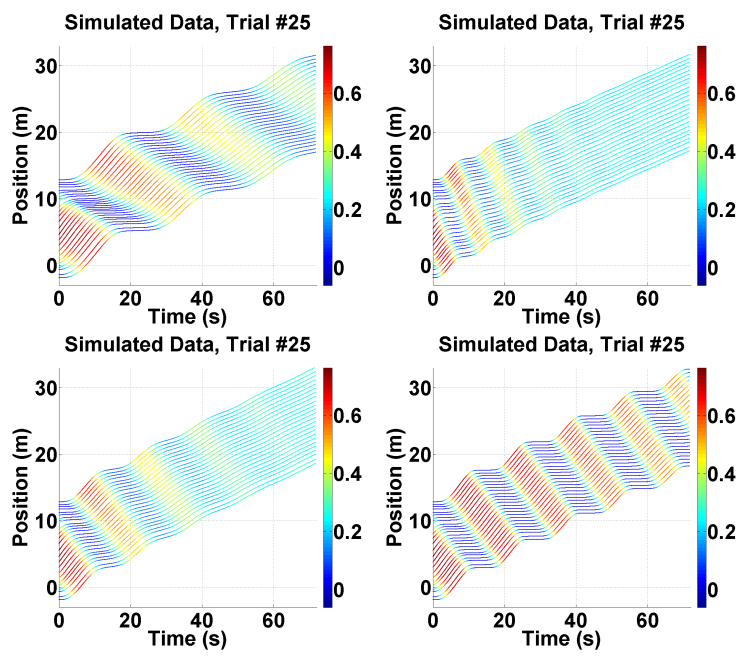

Figure 11: Based on the initial conditions of trial \# 25, simulations are run again with lower C value (top-left), higherC value (top-right), lower $\tau$ value (bottom-left) higher $\tau$ value (bottom-right).

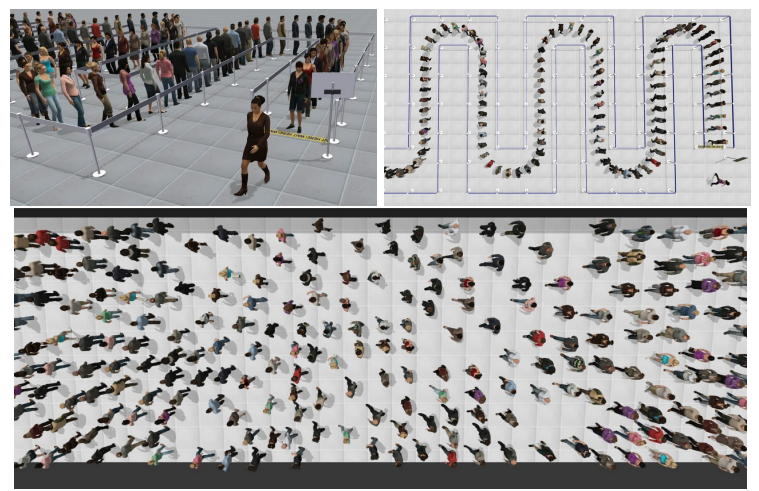

Figure 12: Two application scenarios. First one is a pure $1 D$-traffic situation where virtual walkers are queuing along a winding path (top). Second one is a corridor traffic that combines avoidance and following behaviors (bottom)

It makes it a concurrent solution for collision avoidance as well.

\section{Discussion and Conclusion}

Our approach is based on experimental study. Compared to previous work, our kinematics dataset has been acquired by the mean of optoelectronic motion capture with yet unrivaled precision. Several hypotheses could be compared and a realistic model - where acceleration is controlled as a function of relative speed and distance to the target - is obtained. The properties of macroscopic emergent stop-and-go waves at a higher scale can be measured as well.
Experimental data at a microscopic scale enable the calibration of our model. Experimental data analysis at a macroscopic scale enable evaluating our model. Simulated data fit real trajectories at these two scales. Results are compared to previous approaches to demonstrate improvements. One quality of our model is to fit a range of densities and to enable the emergence of stop-and-go waves in this range. By contrast, some previous models need to be tuned for each density and some fail to simulate stop-and-go waves.

Our model can be more generally used as a speed control law when locomotion is constrained by an obstacle in the sagittal plane. This is particularly emphasized by our corridor scenario: we decompose locomotion control and adapt speed and orientation independently. Speed is controlled by our model while orientation is controlled, in this example, by a simple overpassing rule: believable results are obtained and the emergence of stop-and-go waves is preserved. Note that this idea of decoupling orientation and speed control has already been proposed in previous work [OPOD10].

We were able to provide a unique calibration of parameters to fit the model with the microscopic real data and the fundamental diagram, but had to recalibrate the model to fit the properties of real stop-and-go waves at the best. This reveals how difficult the problem of coupling micro and macroscopic behaviors is. Nevertheless, this question opens the most interesting future work directions.

A first direction is to work again our model formulation: it fits $77.4 \%$ of our experimental data. Do we need to identify how human control their motion in the remaining $22.6 \%$ ? Is the instability problem we mentioned solved by the behavior of walkers during these phases out of the validity domain? It also seems our model is more adapted to some range of density. Should some secondary term be added to the model to cover wider density range?

A second direction is to revisit our calibration technique. We considered $\gamma$ to be constant and unique, this assumption could be reconsidered. We also did not deeply explored parameters interdependencies.

A third direction is to independently consider situations where the target relative speed is positive or negative, i.e., when it is coming closer or going away. The absence of reaction in the unconstrained case (going away) does not cause any danger: there is no urgent need to re-accelerate. Nevertheless, in the example of Figure 3, it seems that participants still behave the same in constrained and unconstrained situations. Deeper analysis is required.

As a conclusion, we propose a model for simulating following behaviors that matches the requirement of crowd simulation. We show that following behavior is probably prominent in unidirectional pedestrian traffic. Moving crowds are complex systems the mechanics of which is particularly difficult to decipher. In this paper, we focus on the emergence of stop-and-go waves which are observed in uni- 
directional traffic. We observed this phenomenon in laboratory experiments and explain these patterns from a specific type of interaction. Based on a new numerical model of following behaviors, our simulations reproduce the observed phenomena both at macroscopic and microscopic levels. We finally open future research paths toward coupling micro and macroscopic behaviors.

\section{Acknowledgments}

This work has been funded by French research agency ANR via PEDIGREE project ANR-08-SYSC-015. Experiments were performed by the PEDIGREE partnership at the University of Rennes 1 , with the help of the laboratory M2S from the University of Rennes 2. We are in particular grateful to A. Crétual, A. Marin and A.-H. Olivier for their help during the experiments.

\section{References}

[AKMR02] Aw A., Klar A., Materne T., RAscle M.: Derivation of continuum traffic flow models from microscopic follow-the-leader models. SIAM Journal on Applied Mathematics 63, 1 (2002), pp. 259-278. 2, 3, 6

[BMdOB03] Braun A., Musse S. R., De Oliveira L. P. L., BODMANN B. E. J.: Modeling individual behaviors in crowd simulation. Computer Animation and Social Agents, International Conference on 0 (2003), 143. 2

[CSC09] Chattaraj U., Seyfried A., Chakroborty P.: Comparison of pedestrian fundamental diagram across cultures. Advances in Complex Systems 12 (2009), 393-405. 2

[DH03] DAAMEN W., Hoogendoorn S. P.: Qualitative results from pedestrian laboratory experiments. In Pedestrian and evacuation dynamics (2003), pp. 121-132. 2

[Faj07] FAJEN B.: Affordance-based control of visually guided action. Ecological Psychology 19, 4 (2007), 383-410. 2

[GCC*10] Guy S. J., Chhugani J., Curtis S., Dubey P., LiN M., MANochA D.: Pledestrians: a least-effort approach to crowd simulation. In Proc. ACM SIGGRAPH/Eurographics Symp. on Computer Animation (2010), pp. 119-128. 8

[GCK*09] Guy S. J., Chhugani J., Kim C., Satish N., Lin M., MANOCHA D., DUBEY P.: Clearpath: highly parallel collision avoidance for multi-agent simulation. In Proc. ACM SIGGRAPH/Eurographics Symp. on Computer Animation (2009), pp. $177-187.2$

[HFV00] Helbing D., FARKAS I., VicseK T.: Sim. dynamical features of escape panic. Nature 407 (2000), 487-490. 2, 8

[JHS07] Johansson A., Helbing D., Shukla P. K.: Specification of the social force pedestrian model by evolutionary adjustment to video tracking data. Advances in Complex Systems 10, supp02 (2007), 271-288. 8

[KGS06] Kretz T., Grünebohm A., Schreckenberg M.: Experimental study of pedestrian flow through a bottleneck. Journal of Statistical Mechanics: Theory and Experiment, 10 (2006). 2

[KO10] Karamouzas I., Overmars M.: Simulating the local behaviour of small pedestrian groups. In Proc. ACM Symp. on Virtual Reality Software and Technology (2010), pp. 183-190. 2
[Lee76] LEE D. N.: A theory of visual control of braking based on information about time-to-collision. Perception 5, 4 (1976), 437-459. 2

[LJC01] LI T.-Y., JENG Y.-J., CHANG S.-I.: Simulating virtual human crowds with a leader-follower model. In Proc. Computer Animation. (2001), pp. $93-102.2$

[LMM03] Loscos C., Marchal D., Meyer A.: Intuitive crowd behaviour in dense urban environments using local laws. Theory and Practice of Computer Graphics 0 (2003), 122. 2

[LMM*11] Lemercier S., Moreau M., Moussaïd M., Theraulaz G., Donikian S., Pettré J.: Reconstructing motion capture data for human crowd study. In Motion in Games. 2011, pp. 365-376. 4

[MT01] Musse S., Thalmann D.: Hierarchical model for real time simulation of virtual human crowds. Visualization and Computer Graphics, IEEE Trans. on 7, 2 (2001), 152 -164. 2

[OPOD10] OndŘEJ J., Pettré J., Olivier A.-H., DONIKIAN S.: A synthetic-vision-based steering approach for crowd simulation. ACM SIGGRAPH 2010 Papers (2010). 2, 9

[PAB07] Pelechano N., Allbeck J. M., Badler N. I.: Controlling individual agents in high-density crowd simulation. In Proc. ACM SIGGRAPH/Eurographics Symp. on Computer animation (2007), pp. 99-108. 2

[POO*09] Pettré J., OndŘEj J., Olivier A.-H., Cretual A., DoniKian S.: Experiment-based modeling, simulation and validation of interactions between virtual walkers. In Proc. ACM SIGGRAPH/Eurographics Symp. on Computer Animation (2009), pp. 189-198. 8

[Rey87] REYNOLDS C. W.: Flocks, herds and schools: A distributed behavioral model. In SIGGRAPH '87: Proc. Computer graphics and interactive techniques (1987), pp. 25-34. 2

[Rey99] REYNOLDS C.: Steering behaviors for autonomous characters. In Game Developers Conference (1999). 2, 8

[Sch01] ScHADSCHNEIDER A.: Cellular automaton approach to pedestrian dynamics - theory. In Pedestrian and evacuation $d y$ namics (2001), pp. 75-86. 2

[SSKB05] Seyfried A., StefFen B., Klingsch W., Boltes M.: The fundamental diagram of pedestrian movement revisited. Journal of Statistical Mechanics: Theory and Experiment 10 (2005). 2, 5

[ST07] SHAO W., TERZOPOUlOS D.: Autonomous pedestrians. Graphical Models 69, 5-6 (2007), 246 - 274. 2

[TCP06] Treuille A., CoOper S., Popović Z.: Continuum crowds. In ACM SIGGRAPH 2006 Papers (2006), pp. 11601168. 2

[Wei93] WEIDMANN U.: Transporttechnik der Fussgänger Transporttechnische Eigenschaften des Fussgängerverkehrs (Literaturstudie). Tech. rep., Institut füer Verkehrsplanung, Transporttechnik, Strassen- und Eisenbahnbau IVT an der ETH Zürich, 1993. in German. 5

[Yam98] YAMORI K.: Going with the flow : Micro-macro dynamics in the macrobehavioral patterns of pedestrian crowds. Psychological review 105 (1998), 530-557. 2

[YW95] YILMAZ E. H., WARREN W. H.: Visual control of braking: A test of the $\tau$ hypothesis. Journal of Experimental Psychology: Human Perception and Performance 21, 5 (1995), 9961014. 2 